\title{
Método ACMANT (Adapted Caussinus-Mestre Algorithm for homogenising Networks of Temperature series): aplicação em dados de Temperatura média do ar da cidade de Aracaju-SE (Brasil)
}

\author{
Method ACMANT (Adapted Caussinus-Mestre Algorithm for homogenising \\ Networks of Temperature series): application in mean air temperature data from the \\ city of Aracaju-SE (Brazil)
}

\author{
Alexandre B. Lopo ${ }^{1}$, Peter Domonkos², Maria Helena C. Spyrides ${ }^{3}$, \\ Javier Sigró ${ }^{4}$, Paulo. S. Lucio ${ }^{5}$
}

\begin{abstract}
${ }_{1}^{1}$ Programa de Pós-Graduação em Ciências Climáticas, Universidade do Estado da Bahia, Bahia - Brasil
${ }^{2,4}$ Centre for Climate Change (C3) Campus de les Terres de l’Ebre, University Rovira I Virgili, Tortosa - Spain

${ }^{3,5}$ Programa de Pós-Graduação em Ciências Climáticas, Departamento de Estatística, Universidade do Estado da Bahia, Bahia - Brasil
\end{abstract}

\begin{abstract}
Resumo
A homogeneização é um processo para aperfeiçoar a qualidade dos dados. Em climatologia, a homogeneização analisa e ajusta tendências temporais de variáveis climáticas, causadas por fatores não climáticos. O objetivo deste trabalho foi realizar a homogeneização de dados da Temperatura média do ar (Tma) aplicando o método ACMANT (Adapted Caussinus-Mestre Algorithm for homogenising Networks of Temperature series) e analisar tendências da Tma em Aracaju-SE. O método ACMANT é totalmente automático em código Fortran e utiliza séries temporais em relação múltipla para o processo de homogeneização. O ACMANT é um aperfeiçoamento do método Caussinus-Mestre, que consiste em uma das ferramentas mais eficazes entre os métodos de homogeneização conhecidos. A metodologia utilizou a versão ACMANTv1.2 e dados mensais de Tma do período 1961-2012 da capital de Sergipe (Aracaju) e cidades próximas obtidas no Banco de Dados Meteorológicos para Ensino e Pesquisa (BDMET), a Normal climatológica do Instituto Nacional de Meteorologia do Brasil (INMET) e teste Mann-Kendall para a análise não-paramétrica da tendência da série de Tma. Os dados originais tinham 23,7\% de falhas que foram preenchidas pelo método. Os resultados do Teste Mann-Kendall, após a homogeneização, mostraram tendências significativas (valor-p < 5\%) de elevação da Tma e aumento de $0,5^{\circ} \mathrm{C}$ na última década (2000 a 2010) em relação a Normal Climatológica de $26^{\circ} \mathrm{C}$ do INMET.
\end{abstract}

Palavras-chave: homogeneização de séries temporais, teste Mann-Kendall, dados ausentes, Normal Climatológica, elevação da temperatura média do ar.

\begin{abstract}
The homogenization is a procedure to improve the quality of data. In climatology, homogenization examines and adjusts temporal biases of climatic variables, caused by non-climatic factors. The objective of this study was to homogenize the data mean air temperature (Tma) applying the method ACMANT (Adapted Caussinus-Mestre Algorithm for homogenising Networks of Temperature series) and study trends of Tma in Aracaju-SE. ACMANT is a fully automatic in Fortran code that uses multiple relative time series for homogenization. The ACMANT is a further development of the Caussinus-Mestre method, that is one of the most effective tool among the known homogenising methods. The methodology of this study used ACMANTv1.2 version and monthly data of Tma for the period 1961-2012 to capital of Sergipe (Aracaju) and eight nearby cities obtained in Meteorological Database for Education and Research (BDMEP), the Climate Standard of the National Institute of Meteorology of Brazil (INMET) and application and Mann-Kendall test for non-parametric analysis of trend in the study of Tma series. In the series of original data existed $23.7 \%$ missing value that were filled by the method. The results the Mann-Kendall test, after the homogenization process, showed trends significant (p-value $<5 \%$ ) of elevated to Tma for the period with an increase of $0.5^{\circ} \mathrm{C}$ over the last decade (2000-2010) in reference to Climate Standard INMET of $26^{\circ} \mathrm{C}$.
\end{abstract}




\section{Introdução}

Para realizar estudos de mudanças climáticas, são necessárias séries temporais hidrometeorológicas longas, contínuas e homogêneas, as quais proporcionam grandes vantagens, em razão de fornecer estatísticas representativas que caracterizam os fenômenos climáticos. As instituições nacionais que fornecem informação hidrometeorológica têm seus próprios sistemas de controle de qualidade, mas há a necessidade de uma homogeneização destes dados para a obtenção de um conjunto de estações básicas, representativas do Brasil, para realizar estudos de mudanças climáticas. Apesar de muitos estudos climáticos serem realizados, ainda não foram elaborados estudos da qualidade e confiabilidade dos dados disponíveis em escala nacional, pelo qual não se conta com esta informação em nenhum conjunto de dados base (MARENGO \& VALVERDE, 2007).

Marengo \& Valverde (2007) explicam que as fontes de erro dos dados podem ser classificadas em três categorias: (i) consistência interna; (ii) randômicas e; (iii) de não homogeneidade. Os erros de consistência interna podem resultar, por exemplo, da transposição das observações. Os erros randômicos podem aparecer por defeito de comunicação eletrônica, degradação dos sensores e outras causas, os quais muitas vezes são difíceis de serem percebidos. E, finalmente, os erros de não homogeneidade ocorrem principalmente quando existem mudanças nas práticas observacionais e podem ser detectadas por certas descontinuidades, tendências, etc. no registro dos dados.

Os erros de não homogeneidade podem ser resolvidos através do processo de homogeneização, cujo objetivo é aperfeiçoar a qualidade dos dados. Em climatologia, a homogeneização analisa e ajusta tendências temporais de variáveis climáticas, causadas por fatores não climáticos (ALEXANDERSSON, 1986; PETERSON \& EASTERLING, 1994).

Nas últimas décadas, grande número de métodos de homogeneização foi desenvolvido, sendo que a ação Advances in homogenisation methods of climate series: an integrated approach HOME (COST ES 0601), realizada entre 2007 e 2011, acelerou o desenvolvimento e validação de métodos como o CLIMATOL, ACMANT e HOMER (HOMOgeinização em softwarE R), disponível em www. homogenisation.org/, sendo que o método HOMER funciona em código aberto R (R Development Core Team, 2012) juntamente com o método SNHT - Standard Normal Homogeneity Test (VINCENT et al, 2002, VENEMA \& MESTRE, 2010; DOMONKOS, 2013; MESTRE et al, 2013).

Nesses métodos é aplicada uma ampla gama de ferramentas estatísticas para a detecção em séries meteorológicas de pontos de mudanças (change-points em inglês) ou de tendências de origem não climática associadas com a falta de homogeneização (IH) (DOMONKOS, 2011).

As principais ferramentas de detecção de IH são: (a) exame de anomalias acumuladas (CAUSSINUS \&
MESTRE, 2004), (b) estatísticas de classificação de ordem (MANN \& WHITNEY, 1947; LANZANTE, 1996), (c) regressão linear múltipla (SOLOW, 1987; VINCENT, 1998), (d) testes $t$ (ALEXANDERSSON, 1986; WANG et al, 2007), (e) análise múltipla com teste de Fisher (SZENTIMREY, 1999) e (f) função degrau adaptada (CAUSSINUS \& LYAZRHI, 1997).

Além disso, outros métodos de homogeneização foram aplicados por Peterson et al (1998), Aguilar et al (2003), Ducré-Robitaille et al (2003); Auer (2005), Brunet et al, (2006) e Menne \& Williams (2005, 2009), entretanto ainda apresentam incertezas sobre sua eficiência.

No presente artigo foi aplicado um método de homogeneização recentemente desenvolvido, denominado ACMANT, abreviação para Adapted Caussinus-Mestre Algorithm for homogenising Networks of Temperature series. Este método apresenta algumas características absolutamente novas que podem influenciar positivamente a eficácia da homogeneização. A inovação mais importante no ACMANT é a utilização de testes de duas variáveis (bivariate detection) para detectar $\mathrm{IH}$ em séries mensais de temperaturas do ar (mínimas, médias e máximas) e precipitação acumulada. As duas variáveis de deteç̧ão são a média anual e a amplitude do ciclo sazonal. Essas variáveis têm frequentemente pontos de mudança simultâneos considerando-se que o tamanho da IH em séries de temperatura apresentam ciclo sazonal de grande amplitude (DOMONKOS, 2006; BRUNET et al, 2011).

Este artigo descreve brevemente o funcionamento de todo o processo de homogeneização via método ACMANT, discute suas características e propriedades gerais e realiza uma aplicação em dados reais de temperatura média do ar (Tma) para a cidade de Aracaju-SE. Em sequência realiza um estudo de tendência da série homogeneizada da Tma.

O estudo aplicou o método ACMANT na variável Tma considerando sua importante na descrição do clima de um local ou região. De fato, todos os processos biológicos e todas as atividades humanas são influenciados decisivamente pela temperatura do ar, cujos valores extremos condicionam a possibilidade da vida animal e vegetal, além disso, a Tma consiste em um dos parâmetros utilizados para o monitoramento das alterações climáticas em relação ao aquecimento global (MEIRELLES \& VASCONCELOS, 2011).

\section{Material e métodos}

A metodologia desse estudo utilizou dados mensais de Tma do período de 1961 a 2012 da cidade de AracajuSergipe e de estações vizinhas.

Para a homogeneização e o preenchimento de falhas foram utilizadas nove séries históricas de postos vizinhos à estação de $\mathrm{n}^{\mathbf{0}} \mathbf{8 3 0 9 6}$ do Instituto Nacional de Meteorologia do Brasil (INMET) de Latitude: -10.95, Longitude 
-37.04 e altitude de 4,72 m localizada em Aracaju. Os dados de Tma foram obtidos no Banco de Dados Meteorológicos para Ensino e Pesquisa (BDMEP/INMET), e as Normais Climatológicas no INMET (RAMOS; DOS SANTOS, 2009).

As nove séries históricas de postos vizinhos à estação de Aracaju são: Itabaianinha e Propriá, em Sergipe; Água Branca, Pão de Açúcar, Porto de Pedras, Palmeira dos Índios e Maceió, no estado de Alagoas; Serrinha e Salvador, no estado da Bahia.

Aos dados foi aplicada a versão ACMANT versão 1.2, disponível em www.c3.urv.cat/members/pdomonkos. html.

Para o estudo de tendências dos dados anuais foi aplicada a análise não paramétrica de Mann-Kendall, com nível de significância de 5\%.

\subsection{Aréa de estudo}

O município de Aracaju, capital do estado de Sergipe, está localizado na região Nordeste do Brasil, inserido na mesorregião do Leste Sergipano, e abrange uma área de $181,8 \mathrm{~km}^{2}$.

A cidade possui Clima Tropical Nordeste Oriental (ARAÚJO et al, 2010), com temperatura do ar considerada quente (média $\geq 18^{\circ} \mathrm{C}$ em todos os meses do ano) e úmido (três meses secos), como se observa na Figura 1 (BRASIL, IBGE, 2013). A proximidade em relação ao oceano e às interações de atuação dos sistemas meteorológicos durante o ano resulta no domínio do clima úmido, com regime pluviométrico do tipo mediterrâneo, definindo-se um período seco de primavera-verão e um período chuvoso de outono-inverno.

A temperatura do ar de Aracaju apresenta máximas absolutas elevadas com $39,9^{\circ} \mathrm{C}$ registrados no mês de fevereiro, e $34,2^{\circ} \mathrm{C}$ em março (ARAÚJO et al., 2010). A Normal climatológica anual da temperatura média do ar é $26,0^{\circ} \mathrm{C}$, com mínima de $23,2^{\circ} \mathrm{C}$ e temperatura máxima $28,5^{\circ} \mathrm{C}$ (RAMOS; DOS SANTOS, 2009).

\subsection{ACMANT}

O método ACMANT proporciona de forma automática uma homogeneização estatística de alta qualidade para dados (mensais ou diários) de temperatura do ar e precipitação. $\mathrm{O}$ método consiste de um procedimento de homogeneização relativa, sendo necessário para a sua aplicação um mínimo de quatro estações ou séries climáticas que estejam correlacionadas espacialmente. O software realiza o cálculo de correlações espaciais (DOMONKOS, 2011).

O programa em FORTRAN é composto por dez arquivos (DOMONKOS, 2011):

i) ACMAN2TmTxmth.exe: arquivo executável que realiza o processo de homogeneização de médias mensais de temperaturas médias ou máximas do ar;

ii) ACMAN2TmTxday.exe: arquivo executável que realiza o processo de homogeneização de valores diários de temperaturas médias ou máximas do ar;

iii) ACMAN2Tminmth: arquivo executável que rea-
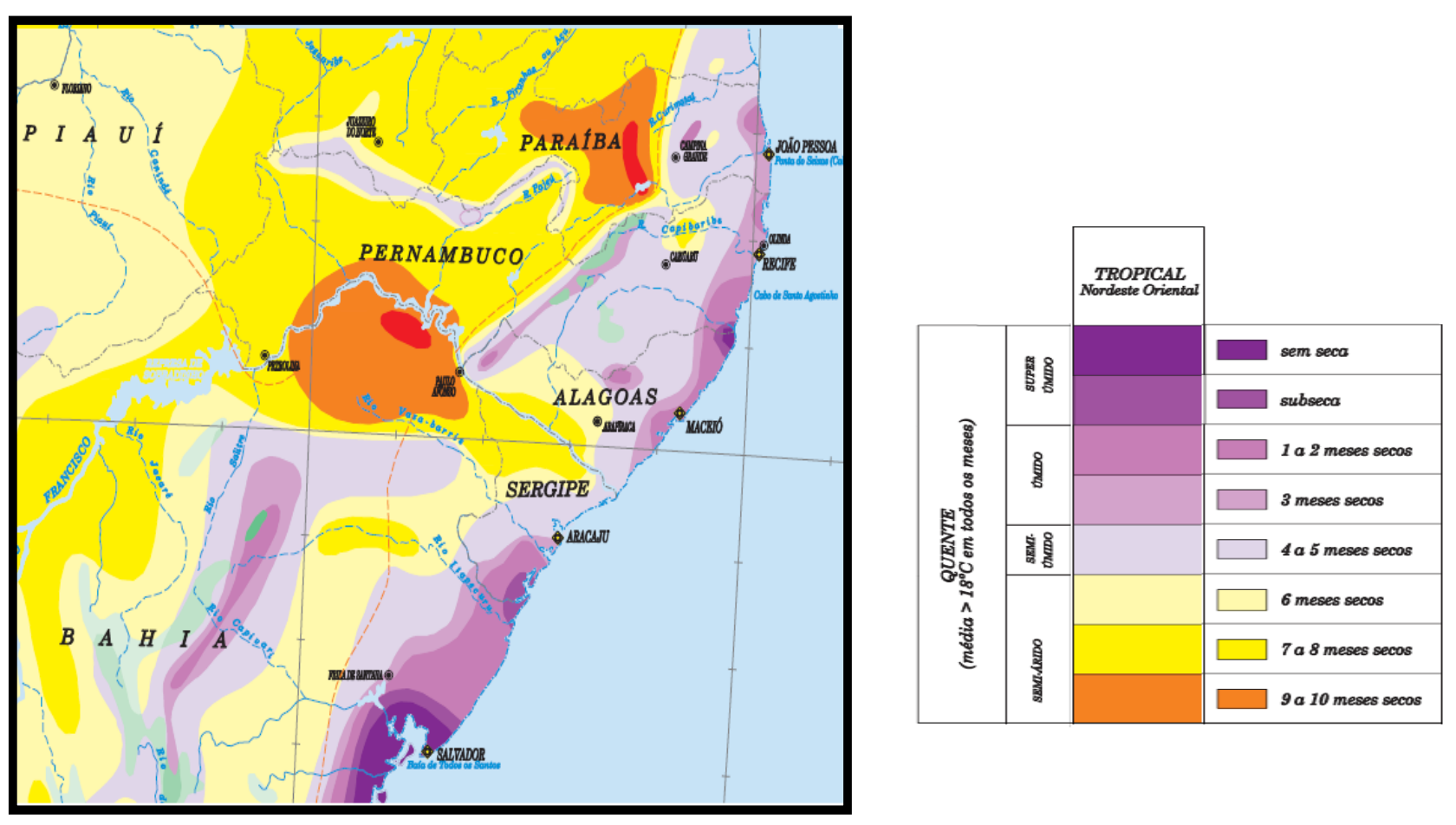

Figura 1 - Clima de Sergipe e de parte do Nordeste. Legenda abaixo indica a classificação do Clima Tropical Nordeste Oriental correspondente à cidade de Aracaju. Fonte: Adaptado de Mapa de Clima do Brasil (BRASIL, IBGE, 2013) 
liza o processo de homogeneização médias mensais de temperaturas mínimas do ar;

iv) ACMAN2Tminday: arquivo executável que realiza o processo de homogeneização para valores diários de temperaturas mínimas do ar;

v) ACMANPrecmth: arquivo executável que realiza o processo de homogeneização de precipitação acumulada mensal;

vi) ACMANPrecday: arquivo executável que realiza o processo de homogeneização de precipitação acumulada diária.

vii) Rtcheck.exe: arquivo executável que realiza o cálculo da correlação espacial das temperaturas mensais;

viii) Rrcheck.exe: arquivo executável que realiza o cálculo da correlação espacial da precipitação acumulada mensal (trabalha com valores de precipitação transformados);

ix) attab.dat: arquivo suplementar para o processo de homogeneização;

x) ACMANTv2.1_Manual.doc: guia para uso.

\subsubsection{Caracterização geral}

São três as características mais importantes do ACMANT: (i) harmonização dos testes em diferentes escalas de tempo (anuais e mensais); (ii) uso do critério Caussinus-Lyazrhi (CAUSSINUS \& LYAZRHI, 1997) na detecção de IH; (iii) uso de análise de variância (ANOVA) para as correções finais da IH (CAUSSINUS \& MESTRE, 2004; DOMONKOS, 2011).

ACMANT é um método de homogeneização automático. Isso significa que depois de introduzir os dados brutos e algumas características da rede (número de estações, resolução temporal das séries, etc) a execução não necessita de assistência humana, sendo que os dados homogeneizados finais são produzidos pelo software (DOMONKOS, 2011; CAUSSINUS \& MESTRE, 2004). É recomendado para séries de temperatura do ar em médias e altas latitudes devido a possuir um algoritmo que supõe ciclo anual quase harmônico de amplitude considerável na IH (DOMONKOS, 2011; CAUSSINUS \& MESTRE, 2004). Em razão dessa recomendação, realizouse neste estudo a homogeneização da Tma por meio da função ACMAN2Tminmth, destinada para temperatura mínima, devido essa função possuir uma amplitude térmica maior e similar as ocorridas em baixas latitudes.

A aplicação do ACMANT pode ser dividida em quatro etapas principais: (I) Preparação: nesta parte são realizados os cálculos iniciais de anomalias, correlações espaciais, filtragem de valores espúrios (outlier) e preenchimento de dados. (II) Pré-homogeneização: esta etapa visa filtrar os erros maiores das séries de referência para realizar homogeneização final. Nesta etapa os ajustes temporários são aplicados para reduzir os efeitos da estação, assim a filtragem de valores espúrios e interpolações são realizadas novamente usando os dados melhorados. (III) Homogeneização: neste item inicialmente se pesquisa as tendências de longo prazo em escala anual (Detecção Principal), em seguida, são determinados os intervalos de pontos de mudança em escala mensal. A Detecção Secundária verifica os efeitos das demais estações em escala mensal. Uma vez que a detecção for concluída, a análise de variância (ANOVA) é aplicada para o cálculo dos termos de correção. A ANOVA também fornece os cálculos finais para preencher os dados ausentes ou os valores atípicos espúrios detectados. (IV) Ajustes finais: aqui os pontos de mudança com desvio e tamanhos insignificantes são excluídos e ANOVA é aplicado novamente com o conjunto reduzido (DOMONKOS, 2011; CAUSSINUS \& MESTRE, 2004; BRUNET et al, 2011).

\subsubsection{Segmentos selecionados de ACMANT}

\subsubsection{Construção da série de referência a partir de grupos de referência:}

Uma série de tempo relativo ( $\mathrm{t}$ ) é a diferença aritmética entre a série candidata $(\mathrm{A})$ e uma série de referência (F). A maneira tradicional de criar séries de referência (PETERSON \& EASTERLING, 1994) é aplicada com uma parametrização específica em ACMANT. Para uma série candidata $(\mathrm{g})$ todas as outras séries da rede $(s=1 \ldots S)$ são usadas como um grupo de referência quando a correlação espacial (r) com g excede um limiar predeterminado. As séries de referências são ponderadas de acordo com as correlações ao quadrado $\left(\mathrm{r}^{2}\right)$. Em ACMANT o limiar de r é 0,4 , no entanto são esperadas pelo menos duas séries com $r \geq 0,5$. Esses limites são relativamente baixos em algumas recomendações (MENNE \& WILLIAMS, 2005; DOMONKOS, 2011). Na equação $1\left[\mathrm{j}_{1}, \mathrm{j}_{2}\right]$ representam dados de uma estação entre os anos de $j_{1}$ e $j_{2^{\prime}}$ ou seja, a equação 1 pode ser aplicada a qualquer período de tempo de uma série (DOMONKOS, 2011).

$$
\mathrm{F}_{\mathrm{g}\left[\mathrm{j}_{1}, \mathrm{j}_{2}\right]}=\frac{\sum_{\mathrm{s}=1}^{\mathrm{s}} \mathrm{r}_{\mathrm{g}, \mathrm{s}}^{2} \mathrm{~A}_{\mathrm{s}\left[\mathrm{j}_{1}, \mathrm{j}_{2}\right]}}{\sum_{\mathrm{s}=1}^{\mathrm{s}} \mathrm{r}_{\mathrm{g}, \mathrm{s}}^{2}}
$$

\subsubsection{Criação de múltiplas séries temporais rela- tivas:}

ACMANT usa múltiplas séries de tempo relativas, pois o número de séries de referência disponíveis varia de acordo com as partes da série histórica. O comprimento mínimo da série $\mathrm{T}$ é de 30 anos. Três séries $\mathrm{T}$ são sempre construídas: (i) com o maior valor possível de $\Sigma \mathbf{r}^{2}$, (ii) com o ano de início mais remoto, (iii) com o último ano. No entanto, como critérios de (i), (ii) e (iii) podem ser satisfeitos por uma mesma série, o verdadeiro número de série $T$ pode ser menor do que 3 . Por outro lado, mais de três séries $\mathrm{T}$ podem estar envolvidas de acordo com as mudanças no somatório das correlações ao quadrado 
$\left(\sum \mathbf{r}^{2}\right)$ para diferentes seções da série, aqui chamada de série candidata (DOMONKOS, 2011).

\subsubsection{Detecção principal:}

A detecção de IH é realizada em cada uma das diferentes séries analisadas. A função da etapa ótima (Optimal step-function) é construída para duas variáveis anuais, ou seja, a média anual $(t m)$ e amplitude do ciclo sazonal $(t d)$, sendo que os pontos de mudança comuns são pesquisados considerando a distância temporal mínima de três anos entre esses pontos. Outros aspectos da detecção principal são idênticos à detecção do tipo PRODIGE (CAUSSINUS; MESTRE, 2010).

Nas equações 2 e 3 são apresentadas as fórmulas para o cálculo de tm e $t d$ por ano j (DOMONKOS, 2011).

$$
\mathrm{tm}_{\mathrm{j}}=\frac{\sum_{\mathrm{m}=1}^{12} \mathrm{t}_{\mathrm{j}, \mathrm{m}}}{12}
$$

$t_{j}=\frac{t_{j, 5}+t_{j, 6}+t_{j, 7}+0,5 \cdot t_{j, 8}-t_{j, 11}-t_{j, 12}-t_{j, 1}-0,5 t_{j, 2}}{3,5}$

Em seguida, a segmentação ótima de um longo período de anos em $\mathrm{K}+1$ segmentos $\mathrm{L}$ é dada pela equação 4 (DOMONKOS, 2011).

$$
\underbrace{\left[\mathrm{j}_{1}, \ldots \mathrm{j}_{\mathrm{K}}\right]}_{\mathrm{j}_{0}=0, j_{\mathrm{K}+1}=\mathrm{L}} \underset{\mathrm{min}}{\min }=\left\{\sum_{\mathrm{k}=0}^{\mathrm{K}} \sum_{\mathrm{i}=\mathrm{j}_{\mathrm{k}}+1}^{\mathrm{j}_{\mathrm{k}+1}}\left(\mathrm{tm}_{\mathrm{i}}-\overline{\mathrm{TM}_{\mathrm{k}}}\right)^{2}+\mathrm{c}_{\mathrm{o}}^{2}\left(\mathrm{td}_{\mathrm{i}}-\overline{\mathrm{TD}_{\mathrm{k}}}\right)^{2}\right\}
$$

O termo superior denota uma média de tempo para o segmento $\mathrm{k}$. A constante $\mathrm{c}_{\mathrm{o}}$ é 1,414 na versão ACMANTv1.2 (DOMONKOS, 2011).

O número de segmentos é otimizado pelo critério Caussinus-Lyazrhi, como indica a seguir a equação 5 (CAUSSINUS; LYAZRHI , 1997).

$$
\ln \left\{1-\frac{\sum_{k=0}^{K}\left(j_{k+1}-j_{k}\right) \cdot\left[\left(\overline{T M_{k}}-\overline{T M}\right)^{2}+c_{0}^{2}\left(\overline{T D_{k}}-\overline{T D}\right)^{2}\right]}{\sum_{i=1}^{L}\left(t m_{i}-\overline{T M}\right)^{2}+c_{0}^{2}\left(t d_{i}-\overline{T D}\right)^{2}}\right\}+\frac{2 K}{L-1} \ln (L)
$$

\subsubsection{Precisão dos períodos da IHs detectados:}

A detecção principal trabalha em escala de tempo relativamente longa, assim, após a detecção principal, os dias com pontos de mudança são identificados e ocorre a aplicação de 48 meses de janelas simétricas em torno dos intervalos pré-estabelecidos pelos pontos de mudança. Nota-se que dentro da janela apenas um ponto de mu- dança é detectado, uma vez que o comprimento mínimo de segmentos é de três anos para a detecção principal. Numa janela, as funções harmônicas de duas fases são configuradas e o valor ideal é pesquisado, sendo aceito somente uma mudança de fase em um dos 25 meses centrais utilizados em um mínimo de 30. O momento da mudança de fase no ajuste ideal é o momento final do ponto de mudança detectada (DOMONKOS, 2011; CAUSSINUS; MESTRE, 2004).

\subsubsection{Detecção secundária:}

Se, após os ajustes aplicados com a detecção principal, ainda as anomalias acumuladas ultrapassam limites predefinidos é necessária uma detecção secundária na qual os efeitos restantes das estações são verificados em escala mensal. A detecção secundária de IHs de curto prazo faz análises em séries temporais relativas e ajustadas de acordo com os resultados da detecção principal. A série temporal relativa e ajustada é examinada em resolução mensal, e os valores máximos de anomalias acumuladas não podem exceder 60 meses. Esta seção tem duas subseções, em que uma busca o máximo de anomalias acumuladas, e a outra detecta as IHs em torno do intervalo de valor máximo de anomalias acumuladas (DOMONKOS, 2011).

Na detecção secundária de 60 meses de uma longa subsérie de valores mensais devem ser examinados valores em torno do máximo de anomalias acumuladas. Neste passo, a segmentação ideal é aplicada para as médias dos valores mensais, sendo substituídas com funções harmônicas de ciclo anual de 10 meses no mínimo, assim o número de segmentos é maximizada por três para uma subsérie (DOMONKOS, 2011; CAUSSINUS; MESTRE, 2004).

\subsubsection{Correção ANOVA:}

Aplica-se para efetuar a correção final minimizando o desvio padrão dos dados homogeneizados. Pode-se verificar que a ANOVA fornece a estimativa ideal dos termos de correção quando existem as seguintes condições: (i) o sinal climático é idêntico, (ii) o efeito da estação é constante entre dois pontos de mudança conhecidos e adjacentes (CAUSSINUS; MESTRE, 2004).

\subsubsection{Pré-homogeneização:}

No software cada série temporal utilizada é pré-homogeneizada de forma que, no cálculo do dos termos para série s, a série g é excluída do processo quando s está preparada para ser um grupo de referência na segmentação final das séries g (CAUSSINUS; MESTRE, 2004).

Como a série s geralmente participa da homogeneização de todas as outras séries temporais da rede, geralmente n-1 diferentes ações de pré-homogeneização são realizadas em uma série individual de uma rede de 
estações de tamanho n (CAUSSINUS; MESTRE, 2004).

Inicialmente, a ordem da série candidata é definida, sendo ela a série estimada com a pior qualidade até a de melhor homogeneidade. A determinação da ordem baseia-se na estimativa dos efeitos máximos de estações. Esse passo inclui necessariamente alguns critérios arbitrários (DOMONKOS, 2011). Durante a pré-homogeneização não é aplicada a ANOVA em razão da análise de variância fazer uso excessivo das ligações espaciais entre os dados. Na pré-homogeneização os termos de ajustamento temporários são aplicados e calculados com base em uma série temporal relativa e unificada (DOMONKOS, 2011; CAUSSINUS \& MESTRE, 2004).

\subsubsection{Ajustes temporários - Série temporal relati- va e unificada (STRU):}

Uma STRU tem as seguintes propriedades: (i) faz a cobertura do período total em que a homogeneização do conjunto candidato pode ser realizada; (ii) inclui as séries de tempo relativamente inalteradas durante o qual o somatório das correlações ao quadrado $\left(\sum \mathrm{r}^{2}\right)$ é mais alta; (iii) a série principal é completada com outras séries de tempo relativas abrangendo o período integral definido por (i), as séries seguintes são complementadas, (iv) as séries complementadas são ajustadas antes da conclusão de forma que tendências sistemáticas, devido a diferenças dos meios e efeitos espaciais das estações, sejam considerados. Em síntese, como a média de todas as estações regionais $(\mathrm{N})$ não é igual à média de alguns subconjuntos de estações de $\mathrm{Q}(\mathrm{Q}<\mathrm{N})$, na construção de STRU é necessário o tratamento deste viés (DOMONKOS,
2011, 2013; CAUSSINUS; MESTRE, 2004).

A versão inicial do ACMANT testada em experimentos cegos identificou que a principal fonte de erros de tendência nas STRUs está associada a falta de sincronismo entre elas (VENEMA; MESTRE, 2010; DOMONKOS, 2011, 2013).

\section{Resultados}

As Figuras 2 e 3 mostram, respectivamente, os resultados da aplicação do ACMANT na série de dados originais da Tma na resolução temporal mensal e anual para a cidade de Aracaju.

Na série mensal havia $23,7 \%$ de falhas que foram preenchidas e homogeneizadas pelo método ACMANT.

A média anual da Tma da série homogeneizada foi de $26,4^{\circ} \mathrm{C}$ com desvio padrão de $0,36^{\circ} \mathrm{C}$, um pouco superior a média dos dados originais de $26,2^{\circ} \mathrm{C}$ e desvio padrão de $0,38^{\circ} \mathrm{C}$, como mostrou as Figuras 3 a e $3 b$.

Para o estudo da tendência da série homogeneizada da Tma, fez-se o filtro de média móvel de 12 meses para dados mensais (Figura 4a) e para dados anuais uma curva de tendência (Figura 4 b) que mostra elevação da Tma.

Os resultados do teste de tendência de Mann-Kendall indicaram tendências positivas e significativas $(\tau=0,563$ e valor $-\mathrm{p}<5 \%$ ) para a Tma no período do estudo.

A tendência positiva em Tma representou um aumento de $0,5^{\circ} \mathrm{C}$ na última década (2000 a 2010) em relação a Normal Climatológica de $26^{\circ} \mathrm{C}$ do INMET obtida no período de 1961 a 1990.

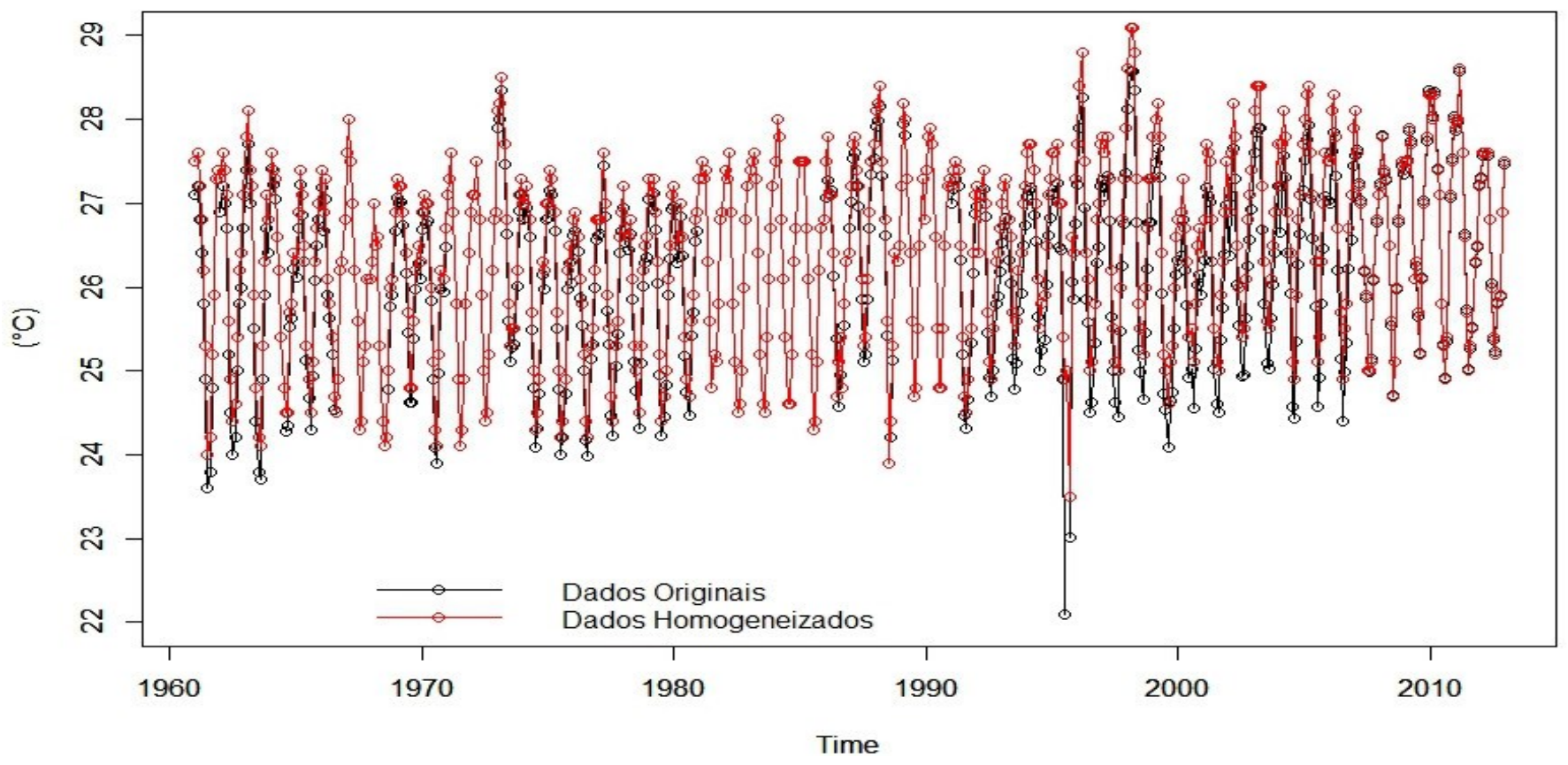

Figura 2 - Série original e homogeneizada da Temperatura média do ar (mensal) para a cidade de Aracaju-SE 

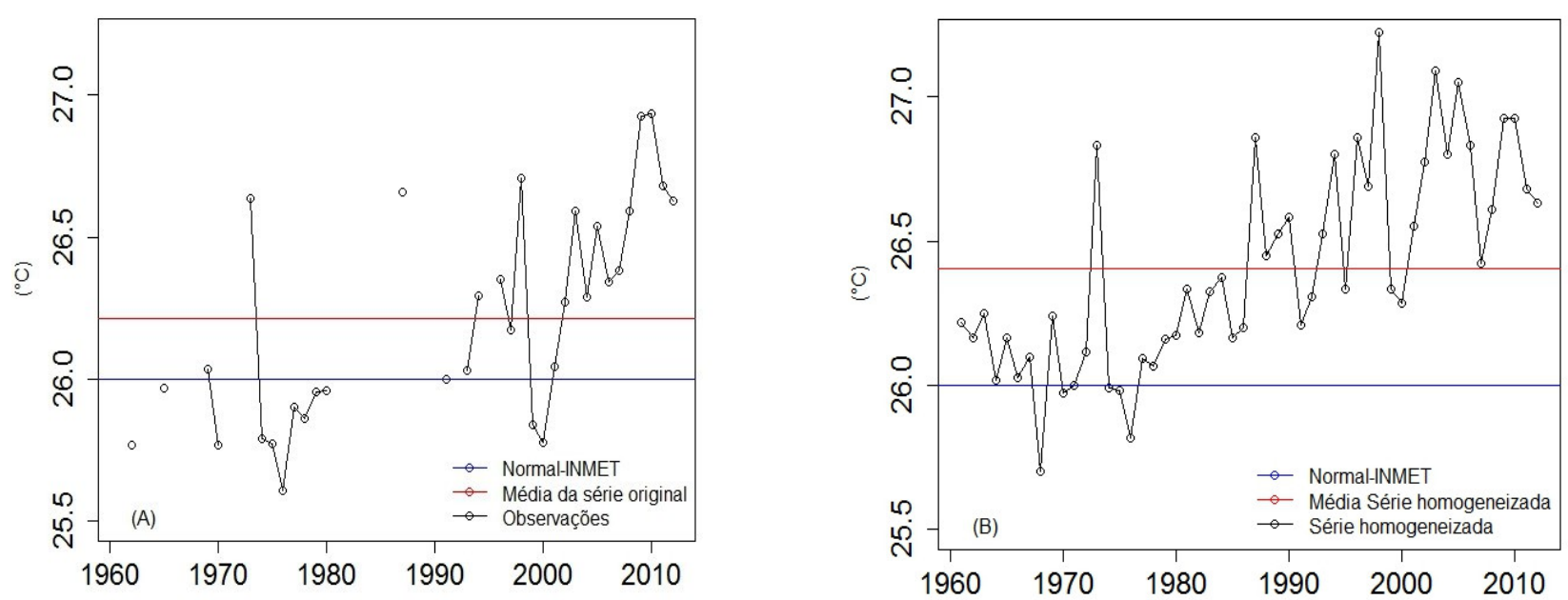

Figura 3 - Temperatura média do ar (escala anual) com indicação da Normal Climatológica (INMET) e média da série para (a) dados originais (b) série homogeneizada pelo método ACMANT. Ambas do período de 1961 a 2012 para a cidade de Aracaju-SE
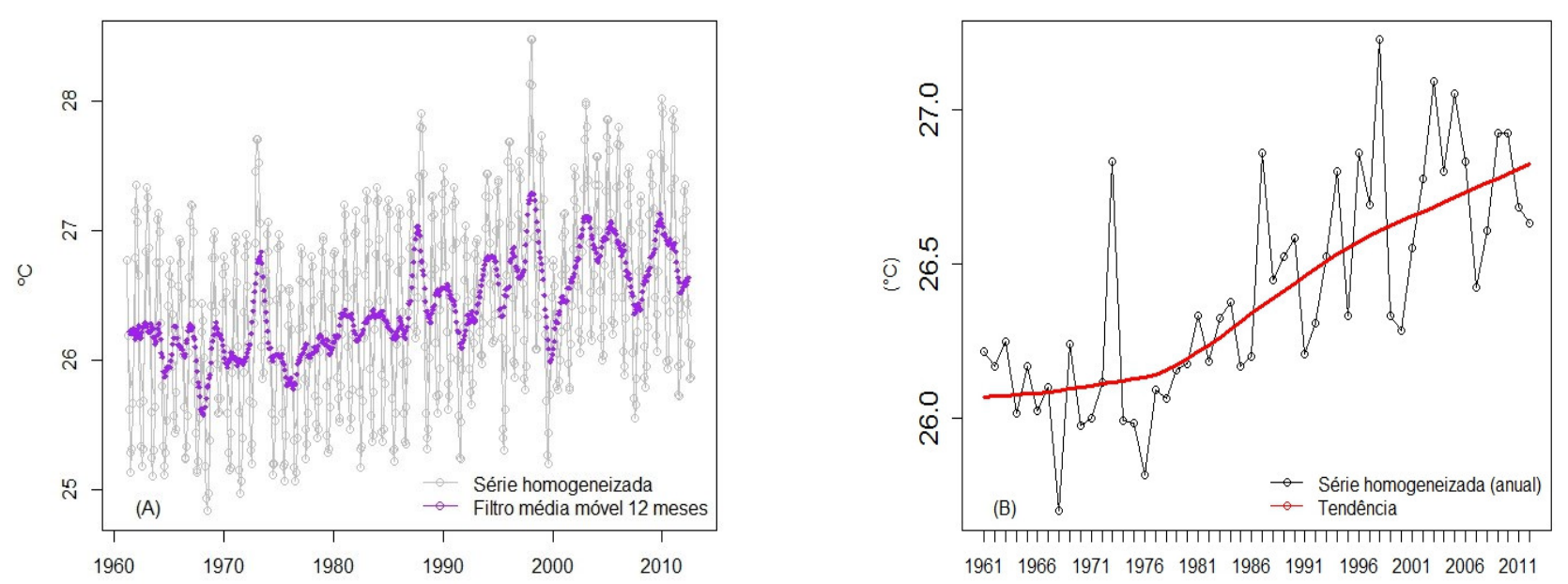

Figura 4 - Série homogeneizada da Temperatura média do ar (mensal) com filtragens médias móveis (a) e dados homogeneizados (anual) com indicação de tendência (b). Ambas do período de 1961 a 2012 para a cidade de Aracaju-SE

\section{Considerações finais}

O método ACMANT aplica um teste bivariado considerando a média anual e a amplitude do ciclo sazonal para identificar os intervalos de falta de homogeneidade.

A rotina do método foi considerada eficiente para a homogeneização dos dados climatológicos de Tma, na medida em que ajustou e completou as lacunas e descontinuidades da série de temperatura com base em parâmetros estatísticos e séries vizinhas. $\mathrm{O}$ algoritmo do método segue quatro etapas sequenciais: preparação, pré-homogeneização, homogeneização e ajustes finais.

No estudo da Tma de Aracaju-SE eliminaram-se as heterogeneidades e lacunas da série do período de 1961 à 2012 visando preservar as peculiaridades da variabi- lidade climatológica da Tma e estudar sua tendência. Dessa forma, o teste Mann-Kendall indicou que há uma tendência significativa e positiva da Tma representando uma elevação em relação à Normal Climatológica (INMET) de $26^{\circ} \mathrm{C}$ na última década.

Por fim, destaca-se a importância da homogeneização de dados para um aperfeiçoamento dos estudos de Climatologia, entre eles as pesquisas sobre tendências em séries temporais.

\section{Agradecimentos}

O presente trabalho foi realizado com apoio do Conselho Nacional de Desenvolvimento Científico e 
Tecnológico (CNPq) através do Doutorado Sanduíche - SWE/CsF (processo 246611/2012-0).

O autor agradece a Universidade Estadual da Bahia pela licença concebida e apoio financeiro durante o Doutorado no Programa de Pós-Graduação em Ciências Climáticas da UFRN e ao Instituto Federal de Educação, Ciência e Tecnologia da Bahia pela licença concebida.

\section{REFERÊNCIAS}

ALEXANDERSSON, H. A Homogeneity Test Applied to Precipitation Data. Journal of Climatology, Vol. 6, No. 6, pp. 661-675, 1986.

AGUILAR, E. et al. WMO Guidelines on Climate Metadata and Homogenization. WMO, Geneva, 2003.

ARAÚJO, H. M. et al. O Clima de Aracaju na Interface com a Geomorfologia de Encostas. 2010.

AUER, I. et al. A New Instrumental Precipitation Dataset for the Greater Alpine Region for the Period 1800-2002. International Journal of Climatology, Vol. 25, No. 2, pp. 139-166, 2005.

BRASIL, IBGE. Mapa de Clima do Brasil. Disponível em <ftp://geoftp.ibge.gov.br/mapas_tematicos/mapas_ murais/>. Acesso. Novembro, 2013.

BRUNET, M. et al. The Development of a New Dataset of Spanish Daily Adjusted Temperature Series (SDATS) (1850-2003). International Journal of Climatology, Vol. 26, No. 13, pp. 1777-1802, 2006.

BRUNET, M. et al. The Minimization of the Screen Bias from Ancient Western Mediterranean Air Temperature Records: An Exploratory Statistical Analysis. International Journal of Climatology, Early View, 2011.

CAUSSINUS, Henri; LYAZRHI, Faouzi. Choosing a linear model with a random number of change-points and outliers. Annals of Institute of Statistical Mathematics, v. 49, n. 4, p. 761-775, 1997.

CAUSSINUS, H.; MESTRE, O. Detection and Correction of Artificial Shifts in Climate Series. Journal of Royal Statistics Society Series C, Vol. 53, No. 3, pp. 405-425, 2004.

DOMONKOS, P. Application of Objective Homogenization Methods: Inhomogeneities in Time Series of Temperature and Precipitation. Időjárás, Vol. 110, pp. 63-87, 2006.

DOMONKOS, Peter. Adapted Caussinus-Mestre Algorithm for Networks of Temperature Series (ACMANT). International Journal of Geosciences, v. 2, n. 3, 2011.
DOMONKOS, Peter. Measuring performances of homogenization methods. Időjárás, v. 117, n. 1, p. 91112,2013

DUCRÉ-ROBITAILLE, J.F., VINCENT L. A, BOULET, G. Comparison of Techniques for Detection of Discontinuities in Temperature Series. International Journal of Climatology, Vol. 23, No. 9, pp. 1087-1101, 2003.

LANZANTE, J. R. Resistant, Robust and Non-Parametric Techniques for the Analysis of Climate Data: Theory and Examples, Including Applications to Historical Radiosonde Station data. International Journal of Climatology, Vol. 16, No. 11, pp. 1197-1226, 1996.

MANN, H. B. and WHITNEY, D. R., On a Test of Whether One of Two Random Variables is Stochastically Larger than the other. The Annals of Mathematical Statistics, Vol. 18, No. 1, pp. 50-60, 1947.

MARENGO, Jose A.; VALVERDE, Maria C. Caracterização do clima no Século XX e Cenário de Mudanças do clima para o Brasil no Século XXI usando os modelos do IPCCAR4. Revista Multiciência, v. 8, p. 5-28, 2007.

MEIRELLES, M. e VASCONCELOS, H. Análise da série histórica de temperatura observada na Ilha Terceira: 1865 - 2004. Anais do XI Congresso Nacional de Engenharia do Ambiente. Lisboa, 2011.

MENNE, M. J.;WILLIAMS JR, C. N. Detection of Undocumented Change points Using Multiple Test Statistics and Composite Reference Series. Journal of Climate, Vol. 18, No. 20, pp. 4271-4286, 2005.

MENNE, M. J. e WILLIAMS JR, C. N. Homogenization of Temperature Series via Pairwise Comparisons. Journal of Climate, Vol. 22, pp. 1700-1717, 2009.

MESTRE, Olivier et al. HOMER: a homogenization software-methods and applications. Quarterly Journal of the Hungarian Meteorological Service, v. 117, n. 1, p. 47-67, 2013.

PETERSON T. C; EASTERLING, D. R. Creation of homogeneous composite climatological reference series. International Journal of Climatology, v. 14, p. 671-679, 1994.

PETERSON, T. C et al,. Homogeneity Adjustments of in Situ Atmospheric Climate Data: A Review. International Journal of Climatology, Vol. 18, No. 13, 1998, pp. 14931517.

RAMOS, Andreas Malheiros; DOS SANTOS, Luiz André Rodriques. Normais climatológicas do Brasil 1961-1990: edição revista e ampliada. INMET, 2009. 
R Development Core Team (2012). R: A language and environment for statistical computing. R Foundation for Statistical Computing, Vienna, Austria. ISBN 3-90005107-0, URL http://www.R-project.org/.

SOLOW, A. R.Testing for Climate Change: An Application of the Two-Phase Regression Model. Journal of Climate and Applied Meteorology, Vol. 26, No. 10, pp. 14011405, 1987.

SZENTIMREY, T. Multiple Analysis of Series for Homogenization (MASH). Annals of Second Seminar for Homogenization of Surface Climatological Data, WMO, Geneva, pp. 27-46, 1999.

VENEMA. V., MESTRE, O. and the COST HOME Team, Benchmark Database, EGU General Assembly, Vienna, Austria, 3-7 May, 2010.

VINCENT, L. A. A Technique for the Identification of Inhomogeneities in Canadian Temperature Series. Journal of Climate, Vol. 11, No. 5, pp. 1094-1104, 1998.

VINCENT LA, ZHANG X, BONSAL BR, Hogg WD. Homogenization of daily temperatures over Canada. Journal of Climate 15:1322-1334, 2002.

WANG X. L., WEN. Q. H., WU Y. Penalized Maximal $t$ Test for Detecting Undocumented Mean Change in Climate Data Series. Journal of Applied Meteorology and Climatology, Vol. 46, No. 6, pp. 916-931, 2007. 